CARDIOVASCULAR MEDICINE

\title{
Assessment of coronary artery stents by 16 slice computed tomography
}

\author{
M Gilard, J C Cornily, P Y Pennec, G Le Gal, M Nonent, J Mansourati, J J Blanc, \\ J Boschat
}

Heart 2006;92:58-61. doi: 10.1136/hrt.2004.056010

See end of article for authors' affiliations

.....................

Correspondence to: Dr Martine Gilard, Department of Cardiology, La Cavale Blanche Hospital, 29609 Brest Cedex, France; martine. gilard@chu-brest.fr

Accepted 13 January 2005 Published Online First 21 April 2005

\begin{abstract}
Objective: To analyse coronary stents with multislice spiral computed tomography (MSCT) in comparison with coronary angiography.

Patients and methods: 310 patients referred for conventional coronary angiography underwent MSCT on the next day (16 $\times 0.75 \mathrm{~mm}$ cross section, $420 \mathrm{~ms}$ rotation, $110 \mathrm{ml}$ contrast agent intravenously at $4 \mathrm{ml} /$ s). Two independent blinded reviewers analysed the MSCT.

Results: 143 patients had previous stenting (232 stents) and 190 (82\%) of the 232 stents were detected. Intrastent lumen was interpretable in $126(64 \%)$ of the detected stents. Lumen interpretability depended on stent diameter: for stent diameter $>3 \mathrm{~mm}, 81 \%$ of lumens were interpretable, as against $51 \%$ with $\leqslant 3 \mathrm{~mm}$ stent diameter $(\mathrm{p}<0.001)$. Restenosis detection likewise depended on stent diameter: with small stents $(\leqslant 3 \mathrm{~mm})$, sensitivity and specificity of MSCT were $54 \%$ and $100 \%$, respectively; positive and negative predictive values were $100 \%$ and $94 \%$. For stents with $>3 \mathrm{~mm}$ diameter, corresponding values were $86 \%, 100 \%, 100 \%$, and $99 \%$.

Conclusion: 16 slice MSCT allows analysis of in-stent lumen in about half of all stented angioplasties. It performs better when stent diameter is more than $3 \mathrm{~mm}$ and may offer a non-invasive alternative to conventional coronary angiography for monitoring stented coronary arteries. Technical progress may improve interpretability and hence increase the yield of MSCT in this application.
\end{abstract}

\section{C} oronary artery stenting is the prime means of revascularising coronary stenosis. Despite the introduction of drug eluting stents, intrastent restenosis remains a major issue in follow up. To date, non-invasive assessment techniques have failed to analyse such intrastent restenosis effectively.' Therefore, coronary angiography remains the reference standard for detection. Multislice spiral computed tomography (MSCT) provides good coronary artery visualisation. $^{23}$ The improved temporal and spatial resolution of the 16 detector row may help to evaluate the necessity of invasive coronary angiography in selected patients and even provide an alternative to conventional coronary angiography. ${ }^{3}$ However, only a limited number of studies reported the feasibility of analysing stents by 16 slice MSCT. ${ }^{45}$ The present study sought to evaluate the effectiveness of this new technique for the assessment of stented coronary arteries and the detection of intrastent restenosis.

\section{METHODS \\ Patients}

During the study period, all patients admitted to our centre for coronary angiography (because of symptoms or a positive stress test, cardiomyopathy evaluation, preoperative clearance, or follow up of coronary bypass grafts) were considered for inclusion in this study. Exclusion criteria were renal insufficiency, atrial fibrillation, or iodine allergy. Finally, 143 patients with and 167 patients without a history of coronary stenting were enrolled. MSCT was performed one day after conventional coronary angiography, regardless of the results of coronary angiography. All patients gave their written informed consent.

\section{MSCT protocol}

Sixteen slice MSCT (Mx8000 IDT 16; Philips, Eindhoven, the Netherlands) was used to acquire a volume dataset
$(16 \times 0.75 \mathrm{~mm}$ cross section, gantry rotation time $420 \mathrm{~ms}$, table feed $2.8 \mathrm{~mm} /$ rotation) covering the distance from the carina to the diaphragmatic side of the heart. A longer scan range was used for patients with coronary artery bypass graft. Tube current was $400 \mathrm{~mA}$, with a tube voltage of $120 \mathrm{kV}$. The entire heart was scanned during a single breath hold. Contrast agent $(110 \mathrm{ml})$ was continuously injected at a rate of $4 \mathrm{ml} / \mathrm{s}$. Automated detection of peak enhancement in the aortic root was used for timing of the bolus.

Cross sectional images were reconstructed with a slice thickness of $0.8 \mathrm{~mm}$ at $0.4 \mathrm{~mm}$ intervals. Retrospective gating was used. Axial images at $40 \%, 50 \%, 65 \%, 75 \%$, and $85 \%$ of the RR interval were reconstructed for each patient and analysed for motion artefacts. The images with the least motion artefacts were used for diagnosis.

Two physicians experienced in MSCT, multiplanar reformats, and three dimensional reconstructions by the "volume rendering" technique independently analysed all datasets (143 MSCTs of patients with coronary stents and 167 MSCTs of patients without coronary stents). They were blinded to patient history, to the coronary angiography data, and to the other physician's interpretation. They were asked to state whether a stent was detectable. For the detected stents, the physicians judged whether the lumen was visually interpretable and investigated whether neointimal proliferation was detectable in the form of a dark, 70 (10) Hounsfield units (HU) density rim. ${ }^{6}$

\section{Quantitative coronary angiography}

Six French invasive coronary angiography was performed one day before MSCT. Two orthogonal angiograms were acquired after intracoronary nitrate injection. An independent blinded observer evaluated the angiograms, used as the standard for stent analysis, by quantitative coronary angiography (GE Medical Systems, Buc, France). Intrastent restenosis was 


\begin{tabular}{|ll|}
\hline Table 1 & Patients' \\
\hline Characteristic & \\
\hline Men & $101(71 \%)$ \\
Age (years) & $68(10)$ \\
Hypertension & $76(53 \%)$ \\
Smoker & $57(40 \%)$ \\
Diabetes mellitus & $31(22 \%)$ \\
Hypercholesterolaemia & $65(45 \%)$ \\
Body mass index ( $\left.\mathrm{kg} / \mathrm{m}^{2}\right)$ & $26(4)$ \\
Serum creatinine $(\mathrm{mmol} / \mathrm{l})$ & $80(6)$ \\
Stent location & \\
LAD & $64(27 \%)$ \\
LCx & $63(27 \%)$ \\
RCA & $75(32 \%)$ \\
LM & $24(10 \%)$ \\
SVG & $6(3 \%)$ \\
$\beta$ Blocker & $118(83 \%)$ \\
Heart rate (beats/min) & $63.5(11.7)$ \\
\hline Data are mean (SD) or number $(\%)$. \\
LAD, left anterior descending artery; LCx, left circumflex \\
artery; LM, left main artery; RCA, right coronary artery; \\
SVG, saphenous vein graft. \\
\hline
\end{tabular}

defined as $\geqslant 50 \%$ diameter stenosis on follow up angiography.

\section{Statistical analysis}

Data were expressed as mean (SD) or as proportions. Sensitivity and specificity for intrastent restenosis detection were assessed against the conventional coronary angiography data as a reference. Non-parametric tests were used to compare groups. A probability value of $\mathrm{p}<0.05$ was considered significant. Cohen's $\kappa$ was calculated to determine interobserver and intraobserver variations in restenosis detection. Associations between the size and both detectability and visibility of stents on MSCT were tested by a $\chi^{2}$ test. The strength of the association was evaluated by odds ratios with their $95 \%$ confidence intervals (CIs).

\section{RESULTS}

\section{Clinical characteristics of the study group}

Table 1 summarises clinical data of the 143 patients, in whom 232 stents were analysed (1.6 (1.2) stents/patient). MSCT plus coronary angiography were performed 12 (8) months after stenting (range 0-21 months), with no complications in any patient. One hundred and eighteen patients (83\%) had been taking and continued to take $\beta$ blocker medication, but no additional $\beta$ blockers were administered.

\section{Stent characteristics}

Ten types of stent were studied: Bestent (Medtronic, Minneapolis, Minnesota, USA; $\mathrm{n}=4$ ), GFX (Medtronic;

\begin{tabular}{|cccl}
\hline \multicolumn{3}{l}{ Table 2} & \multicolumn{2}{l}{ Stent analysis } \\
\hline & $\begin{array}{l}\text { Total } \\
\text { stents }\end{array}$ & $\begin{array}{l}\text { Detectable } \\
\text { stents }\end{array}$ & $\begin{array}{l}\text { Interpretable lumen in } \\
\text { detectable stents }\end{array}$ \\
\hline Stent location & & & \\
LAD & 64 & $58(91 \%)$ & $35(55 \%)$ \\
LCx & 63 & $52(82 \%)$ & $28(44 \%)$ \\
RCA & 75 & $52(69 \%)$ & $35(47 \%)$ \\
LM & 24 & $24(100 \%)$ & $22(92 \%)$ \\
SVG & 6 & $6(100 \%)$ & $6(100 \%)$ \\
Stent diameter (mm) & & \\
2.5 & 42 & $35(83 \%)$ & $12(34 \%)$ \\
3.0 & 86 & $69(80 \%)$ & $40(58 \%)$ \\
3.5 & 61 & $51(84 \%)$ & $41(80 \%)$ \\
4.0 & 42 & $34(81 \%)$ & $28(82 \%)$ \\
4.5 & 1 & $1(100 \%)$ & $1(100 \%)$ \\
\hline
\end{tabular}

$\mathrm{n}=31$ ), Driver (Medtronic; $\mathrm{n}=49$ ), Nir (Boston Scientific, Boston, Massachusetts, USA; $\mathrm{n}=5$ ), ExpressII (Boston Scientific; $\mathrm{n}=42$ ), Liberty (Boston Scientific; $\mathrm{n}=8$ ), Taxus (Boston Scientific; $\mathrm{n}=21$ ), Helistent (Hexacath, Rueil-Malmaison, France; $\mathrm{n}=5$ ), Jostent Flex (Jomed, Rangendingen, Germany; $\mathrm{n}=53$ ), and Cypher (Cordis, Miami, Florida, USA; $\mathrm{n}=14$ ).

Stent diameters were distributed as follows: $2.5 \mathrm{~mm}$, $\mathrm{n}=42(18 \%) ; 3.0 \mathrm{~mm}, \mathrm{n}=86(37 \%) ; 3.5 \mathrm{~mm}, \mathrm{n}=6 \mathrm{l}$ (26\%); $4.0 \mathrm{~mm}, \mathrm{n}=42$ (18\%); and $4.5 \mathrm{~mm}, \mathrm{n}=1(0.4 \%)$. Metallic surface area ranged from $11-20 \%$, and strut thickness ranged from $90-139 \mu \mathrm{m}$.

Conventional angiography detected significant (50-90\%) restenosis in 20 stents (9\%). These stents had diameters of $2.5 \mathrm{~mm}(\mathrm{n}=7), 3.0 \mathrm{~mm}(\mathrm{n}=6), 3.5 \mathrm{~mm}(\mathrm{n}=4)$, and $4.0 \mathrm{~mm}(\mathrm{n}=3)$.

\section{Stent imaging by MSCT}

Table 2 summarises the main MSCT findings.

\section{Detectable stents}

Of the 232 analysed stents, only 190 (82\%) were detected by MSCT. Reasons for non-detection were mainly motion artefacts and severe calcifications. Stent size and MSCT detectability were not correlated but detectability and artery were strongly linked $(p<0.05)$, with right coronary stents proving less detectable (table 2 ). There were no false positive detections. Detection sensitivity and specificity were $82 \%$ and $100 \%$, respectively. Interobserver and intraobserver variations, expressed as $\kappa$ values, were 0.95 and 0.96 , respectively.

\section{Intrastent lumen analysis}

The lumen proved interpretable in 126 stents: 53\% of implanted stents, or $64 \%$ of those detected. Lumen interpretability was associated with the stent diameter, being significantly greater in wider stents $(p<0.001)$ (fig 1$)$. Lumen interpretability rate was $81 \%$ in stents $>3 \mathrm{~mm}$ diameter, as compared with $50 \%$ in stents $\leqslant 3 \mathrm{~mm}$ (odds ratio $3.6,95 \%$ CI 1.9 to 6.9$)$. Lumen interpretability in detected stents did not vary significantly from one stent location to another, or from one model of stent to another.

\section{Restenosis analysis}

All 20 stents ( 13 that were $\leqslant 3 \mathrm{~mm}$ and seven $>3 \mathrm{~mm}$ ) presenting coronarographic restenosis were detected on MSCT. The lumen proved interpretable in 13 of these $(65 \%)$. Seven of the 13 with a diameter $\leqslant 3 \mathrm{~mm}(54 \%)$ and six of the seven with a diameter $>3 \mathrm{~mm}(86 \%)$ had interpretable lumens (fig 2). Restenoses were detected by MSCT in all six interpretable $>3 \mathrm{~mm}$ diameter stents and in four of the seven $\leqslant 3 \mathrm{~mm}$ diameter stents. There were no false positive detections of restenosis on MSCT. For small stent restenosis, sensitivity and specificity of MSCT were 54\% (95\% CI $29 \%$ to $77 \%$ ) and $100 \%$ (95\% CI $96 \%$ to $100 \%$ ), respectively, and the positive and negative predictive values were $100 \%$ (95\% CI $65 \%$ to $100 \%$ ) and $94 \%$ (95\% CI $87 \%$ to $97 \%$ ), respectively. For the $>3 \mathrm{~mm}$ diameter stents, sensitivity and specificity were $86 \%$ (95\% CI $49 \%$ to $97 \%$ ) and $100 \%$ (95\% CI $95 \%$ to $100 \%$ ), respectively, and the positive and negative predictive values were $100 \%$ (95\% CI $61 \%$ to $100 \%$ ) and $99 \%$ (95\% CI $93 \%$ to $100 \%$ ), respectively.

\section{DISCUSSION}

The present study analysed 232 stents with the aim of assessing the feasibility of MSCT for the follow up of stented coronary angioplasty. In our study, $82 \%$ of stents were detected on MSCT, of which $64 \%$ were analysable $(53 \%$ of all stents). A stent diameter $>3 \mathrm{~mm}$ was associated with better 


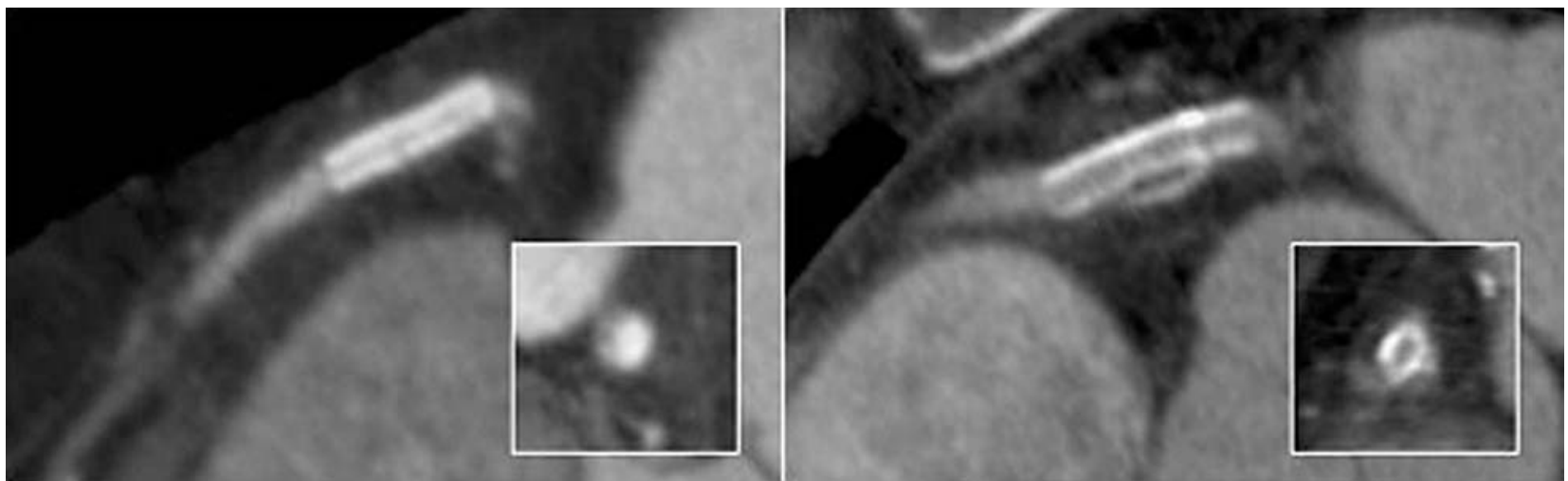

Figure 1 Right coronary artery stents. (A) Stent of $2.5 \mathrm{~mm}$ diameter with a non-interpretable lumen. (B) Stent of $3.5 \mathrm{~mm}$ diameter with an interpretable lumen.

lumen visibility and better ability to detect intrastent restenosis.

Non-invasive detection of intrastent restenosis is a real problem: exercise stress tests, stress echocardiography, and myocardial scintigraphy ${ }^{1}$ have poor sensitivity and specificity, especially in the left main coronary artery. ${ }^{7}$ Coronary angiography is the most dependable means of diagnosis. Stents raise a problem for MSCT analysis: in our series, only 190 of 232 stents (82\%) were detected. Severe calcifications and a right coronary stent location are among the causes of non-detection $(\mathrm{p}<0.05)$, size not being a factor.

\section{Stent lumen analysis}

In vitro studies have analysed stents of different types and diameters by 16 slice MSCT. ${ }^{8}$ The stents produce artefacts by increasing the strut thickness (partial volume artefacts) and thereby artificially reducing the intrastent lumen. These artefacts vary particularly according to the metal being used: the most severe artefacts are found with tantalum or gold coated stents or with covered stent grafts. Moreover, we aimed at analysing the in-stent lumen, whose size is close to the spatial resolution of a computed tomogram. However, as compared with four slice MSCT, 16 slice MSCT allows for decreasing the slices' thickness and for increasing spatial resolution and thus may improve the yield of MSCT in this application.

Kruger et $a l^{10}$ analysed 32 stents in vivo by four slice MSCT. They were unable to interpret the lumen in any of these, whatever the type or diameter of the stent. Restenosis was detected indirectly by distinguishing occluded or permeable stents. This thickening is reduced by $24 \%$ when 16 slice MSCT is used instead of four slice MSCT. ${ }^{9}$ In our study, the model of stent did not affect its detection or the lumen interpretation. The stents used had strut thicknesses $<140 \mu \mathrm{m}$, which in the study of Schuijf et $a l^{5}$ served as a threshold at which struts were deemed thick and more prone to high density artefacts. The determining factor for lumen interpretation was stent size. The interpretability rate rises from $50 \%$ for $\leqslant 3.0 \mathrm{~mm}$ diameters to $81 \%$ when the diameter is $>3.0 \mathrm{~mm}$.
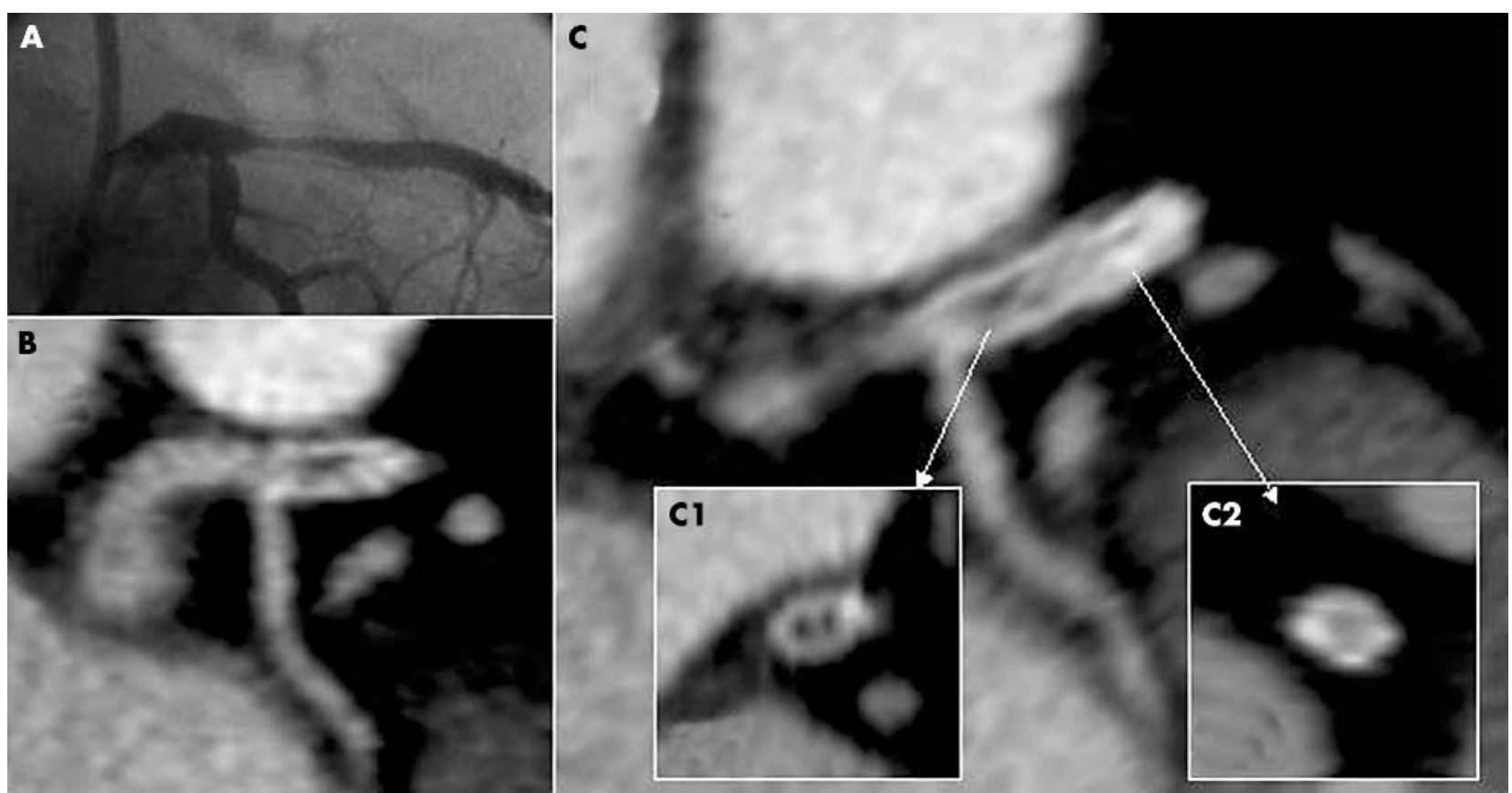

Figure 2 (A) Invasive angiography and (B, C) corresponding multislice spiral computed tomography. $(B, C)$ Longitudinal cross section; $(C 1)$ proximal axial cross section with restenosis; (C2) distal axial cross section without restenosis. 


\section{Intrastent restenosis analysis}

In the case of intrastent restenosis, MSCT lumen analysis shows a larger or smaller rim-like proliferation. ${ }^{6}$ When analysing the density of this hyperplasia by means of measurements taken on several axial sections, the values are found to be in the 70 (10) HU range, which corresponds to intermediate plaques in intracoronary ultrasound terms. ${ }^{11}{ }^{12}$ The HU density of the neointimal hyperplasia approximates that found with a carotid stent: 75.6 (5.6) HU. ${ }^{6}$ Where the lumen is interpretable, restenosis detectability also depends on stent diameter, although these are never false positive. Non-interpretability in our study was due to partial volume effects caused by the stent struts in $\leqslant 3 \mathrm{~mm}$ diameter stents and to motion artefacts and severe calcification in $>3 \mathrm{~mm}$ diameter stents.

Similar to previous studies concerning the assessment of stent patency, in our series the restenosis rate was low. The design of our study, which was not a follow up study but included all patients referred for coronary angiography, may account for this low rate. Therefore, the high negative predictive value for detecting in-stent restenosis has to be interpreted with care.

Use of MSCT as a screening test for patients with previous stenting is appealing. However, this has to be further validated before the use of MSCT can be generalised to routine practice. The amount of extra radiation has to be taken into account in the benefit-risk evaluation of this noninvasive technique. Indeed, in a previous study done by our group, the radiation exposure with 16 slice MSCT was estimated at 12 (5) mSV, as opposed to 4 (2) $\mathrm{mSV}$ for coronary angiography. ${ }^{13}$

\section{Conclusion}

Sixteen slice MSCT allows analysis of the in-stent lumen in about one half of patients with previous coronary artery stenting. Thus, it may be an alternative to conventional coronary angiography, particularly in the follow up of angioplasties with stents $>3.0 \mathrm{~mm}$ in diameter. Nevertheless, calcifications, arrhythmias, high heart rate, partial volume or motion artefacts, and radiation exposure are important limitations of this technique. Technical progress, especially increasing the number of rows, may improve interpretability and hence increase the yield of MSCT in this application.

\section{Authors' affiliations}

M Gilard, J C Cornily, P Y Pennec, J Mansourati, J J Blanc, J Boschat, Department of Cardiology, Brest University Hospital, Brest, France G Le Gal, EA 3878 and Department of Internal Medicine, Brest University Hospital, Brest, France

M Nonent, Department of Radiology, Brest University Hospital, Brest, France

\section{REFERENCES}

1 Zellweger MJ, Weinbacher M, Zutter AW, et al. Long-term outcome of patients with silent versus symptomatic ischemia six months after percutaneous coronary intervention and stenting. J Am Coll Cardiol 2003;42:33-40.

2 Nieman K, Cademartiri F, Lemos PA, et al. Reliable noninvasive coronary angiography with fast submillimeter multislice spiral computed tomography. Circulation 2002;106:2051-4.

3 Ropers D, Baum U, Pohle K, et al. Detection of coronary artery stenoses with thin-slice multi-detector row spiral computed tomography and multiplanar reconstruction. Circulation 2003;107:664-6.

4 Nieaman K, Cademartiri F, Raaijmakers R, et al. Noninvasive angiography evaluation of coronary stents with multi-slice spiral computed tomography. Herz 2003;28:136-42.

5 Schuiif JD, Bax JJ, Jukema W, et al. Feasibility of assessment of coronary stent patency using 16-slice computed tomography. Am J Cardiol 2004;94:427-30.

6 Cademartiri F, Mollet N, Nieman K, et al. Neointimal hyperplasia in carotid stent detected with multislice computed tomography. Circulation 2003; 108:e147.

7 Janosi A, Vertes A. Exercise testing and left main coronary artery stenosis: can patients with left main disease be identified? Chest 1991; 100:227-9.

8 Maintz D, Seifarth H, Flohr T, et al. Improved coronary artery stent visualization and in-stent stenosis detection using 16-slice computedtomography and dedicated image reconstruction technique. Invest Radiol 2003;38:790-5.

9 Mahnken AH, Buecker A, Wildberger JE, et al. Coronary artery stents in multislice computed tomography: in vitro artifact evaluation. Invest Radiol 2004;39:27-33.

10 Kruger S, Mahnken AH, Sinha AM, et al. Multislice spiral computed tomography for the detection of coronary stent restenosis and patency. Int J Cardiol 2003;89:167-72.

11 Schroeder S, Kopp AF, Baumbach A, et al. Noninvasive detection and evaluation of atherosclerotic coronary plaques with multislice computed tomography. J Am Coll Cardiol 2001;37:1430-5.

12 Leber AW, Knez A, Becker A, et al. Accuracy of multidetector spiral computed tomography in identifying and differentiating the composition of coronary atherosclerotic plaques: a comparative study with intracoronary ultrasound. J Am Coll Cardiol 2004;43:1241-7.

13 Gilard M, Cornily JC, Mansourati J, et al. Estimation of radiation exposure with 16-detector row computed tomography of coronary artery. Eur Heart $\mathrm{J}$ 2004;25(suppl):472 [abstract]. 